\title{
Espacios de académicas y su labor en la investigación en Chiapas
}

\section{Academic spaces and their work in research in Chiapas}

\author{
Dra. Danae Estrada Soto \\ Dr. Francisco Iván López Gutiérrez \\ Dra. Yannett Fabiola López Gutiérrez \\ Dra. Gabriela Grajales García \\ Universidad Autónoma de Chiapas \\ danae.estrada@unach.mx \\ ivan.gutierrez@unach.mx \\ yannett.gutierrez@unach.mx \\ gabriela.grajales@unach.mx
}

\section{RESUMEN:}

El presente artículo tiene la finalidad de mostrar un análisis descriptivo de la situación que prevalece en las universidades públicas del estado de Chiapas con respecto de la participación de las académicas principalmente aquellas que están dirigidas a su labor en la investigación en la entidad, cabe destacar que uno de los criterios para considerar como una carrera consolidada en este sentido es su incorporación al Sistema Nacional de Investigadores (SNI) del Consejo Nacional de Ciencia y Tecnología (CONACyT). Esta es la primera etapa de la investigación donde se muestran solo datos que pretenden dar cuenta de las desigualdades de género existentes en al ámbito académico.

Palabras Clave: Género, Educación Superior, desigualdad, academia.

\section{ABSTRAC:}

This article has the purpose of showing a descriptive analysis of the situation that prevails in the public universities of the state of Chiapas with respect to the participation of

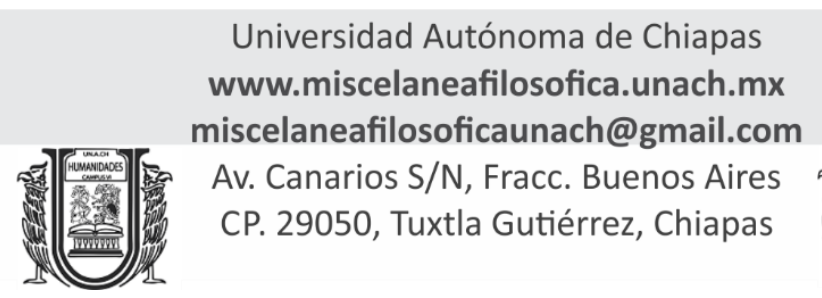


academics, mainly those that are directed to their work in research in the entity, it should be noted that One of the criteria to be considered as a consolidated career in this sense is its incorporation into the National System of Researchers (SNI) of the National Council of Science and Technology (CONACyT). This is the first stage of the research where only data that claim to account for existing gender inequalities in the academic field are shown.

Keywords: Gender, Higher Education, inequality, academia

\section{INTRODUCCIÓN:}

Chiapas es un estado multicultural con marcadas e históricas desigualdades en los ámbitos económico, político, social y educativo; desde la perspectiva educativa, el rezago existente ha sido un tema que se ha tratado en distintos niveles, se ha debatido y analizado desde distintas perspectivas y por distintas investigadoras e investigadores; sin embargo un tema que no se ha considerado de manera recurrente es la participación de las mujeres que se dedican a la investigación y docencia de universidades públicas, en este estado con líneas de acción tan difusas en las que se habla de políticas públicas con perspectiva de género pero que no son aplicadas, donde las académicas se enfrentan a marcadas desigualdades; sin embargo, como veremos más adelante son las que se encargan de la productividad más fuerte en las universidades, a pesar de ser ellas quienes histórica y socialmente se han dedicado además a otras actividades impuestas socioculturalmente.

La reflexión anterior se ha dado a raíz de la tesis de doctorado titulada "Espacios y Tiempos de estudio y trabajo. Experiencias de mujeres académicas en Chiapas" en la que se investigó cuál es la participación de las académicas en los programas de Posgrado pertenecientes al Padrón Nacional de Posgrados de Calidad del Consejo Nacional de Ciencia y Tecnología (PNPC-CONACyT). Esta investigación ha seguido vigente con líneas de investigación que a la fecha se trabajan con otros proyectos de investigación implementados en la Universidad Autónoma de Chiapas (UNACH). 
Actualmente las académicas aún enfrentan marcadas desigualdades en sus espacios laborales, parte de la investigación que actualmente se realiza atiende a la participación de las académicas en las universidades. Para efectos de este artículo se retomará un primer apartado donde se exponga la perspectiva teórica sobre la perspectiva de género en el ámbito académico y un segundo apartado donde se exponga la parte descriptiva, nos situaremos en las mujeres que han logrado acceder al reconocimiento del Sistema Nacional de investigadores (SNI) que otorga en CONACYT, para conocer dicha participación se llevó a cabo un estudio exploratorio que permitió construir una base de datos de seis universidades públicas del estado de Chiapas que cuentan con un importante reconocimiento como lo son: la Universidad Autónoma de Chiapas (UNACH) y de la Universidad de Ciencias y Artes de Chiapas (UNICACH),Universidad Politécnica de Chiapas (UPChiapas), Instituto Tecnológico de Tuxtla Gutiérrez (ITTG), Colegio de la Frontera Sur (ECOSUR), Centro de Investigaciones y Estudios Superiores en Antropología Social (CIESAS).

\section{Perspectiva teórica}

Las temáticas sobre género se han visibilizado y se han debatido desde diferentes instancias internacionales, pero de acuerdo con Bonder (1994) el interés académico, político y social por comprender la situación de las mujeres en la ciencia y la tecnología es reciente, apareciendo algunos estudios en década del 70 y la mayoría de las investigaciones con esta temática aparecieron a mediados de los 90 haciéndose poco a poco más evidentes.

De acuerdo con Bonder (2004), las condiciones de desigualdad entre hombres y mujeres son más notorias en el campo de la ciencia y la tecnología. Es a inicios del siglo XXI que estamos prácticamente al inicio de un reconocimiento internacional de la importancia de esta problemática. En efecto, si bien la IV Conferencia de Naciones Unidas sobre la Mujer realizada en Beijing 1995 constituyó un hito fundamental para dinamizar acciones a favor de la igualdad social de las mujeres de todo el mundo, hay que resaltar que su Plataforma de Acción no contiene un capítulo específico dedicado a la ciencia y la tecnología. Las alusiones y recomendaciones que guardan relación con ello están diseminadas sólo en algunos capítulos, con especial énfasis en educación, economía y pobreza; y sobre todo expresan una preocupación por el acceso 
y participación igualitaria de las mujeres (tanto de las especialistas como el conjunto) a estos recursos de conocimiento (p.7).

Las desigualdades por motivos de género en la ciencia y la tecnología es una situación que se da en muchos países pero que se hace más evidente en América Latina, es importante destacar que muchas políticas públicas incentivadas primordialmente desde los ámbitos educativos suelen ser parte de programas gubernamentales que se terminan al concluir los periodos establecidos de los gobiernos, lo que no da paso a la continuidad que permita líneas fuertes de acción y salir de esta situación que afecta a las mujeres y con ello a distintos sectores sociales.

Actualmente existen congresos y encuentros que se enfocan en analizar la perspectiva de género desde la ciencia y la tecnología, pero hacen falta más debates y estudios que demuestren la importancia de las mujeres en estos sectores y que visibilicen la necesidad de tener a más mujeres en esas áreas, que, aunque exista la capacidad de las mujeres, los factores (socio-culturales) que las envuelven no les permiten colocarse en estas áreas.

El trabajo y el acceso a la educación superior son elementos que abren la posibilidad de cambio y de participación a las mujeres pues el nivel académico significa de cierta forma un estatuto que permite alcanzar otros niveles de consolidación. En el documento de trabajo coordinado por Ortiz-Ortega (2013) para ser comentado en la reunión “Una mirada a la ciencia, tecnología e innovación con perspectiva de género: Hacia un diseño de política públicas" celebrada en la ciudad de México en el año 2013 como parte de los trabajos del Foro Consultivo Científico y Tecnológico del Consejo Nacional de Ciencia y Tecnología se presentaron los siguientes datos:

a) En materia de escolarización se han logrado incrementos significativos:

la población femenina a nivel de educación superior ha crecido de manera ascendente en las últimas tres décadas (...) superando a los hombres en el nivel secundaria (...) la población de mujeres en licenciatura representó (...) en el ciclo escolar 2010-2011 (...) 49\%, y actualmente hay más mujeres que hombres estudiando un posgrado (Ortiz-Ortega, 2013, p. 35). 
b) Pero se presenta una distribución de la matrícula diferenciada por áreas de conocimiento que lleva a las mujeres a elegir preferentemente áreas de conocimiento que tienen relación con roles de cuidadora que tradicionalmente se les asignan. Esta tendencia se presenta en los países miembros de la OCDE en donde en promedio las mujeres obtuvieron $75 \%$ de los grados en el campo de ciencias de la salud y [representaron] más de $70 \%$ de los estudiantes en las carreras de humanidades y educación [en contraste representaron] poco menos de $30 \%$ de los graduados en el campo de las ingenierías, manufacturas y construcción (Ortiz-Ortega, 2013, pp. 35-36).

c) Prevalecen estereotipos culturales que desalientan la participación de las mujeres en la ciencia desde la infancia, estos estereotipos:

señalan que las niñas no tienen la misma capacidad para construir pensamiento abstracto y lógico necesario para las ciencias [o], ante dificultades económicas para mandar a todos los hijos(as) a la escuela primaria (...) tienen a anteponer la educación de los varones (...). Además, generalmente se prepara a las niñas para creer que su futuro será primordialmente el ser madres y no proveedoras económicas del hogar (Ortiz-Ortega, 2013, p. 37).

d) Una vez que han concluido sus estudios profesionales, las mujeres enfrentan mayores dificultades que los hombres para ingresar y permanecer en la comunidad científica. Entre estas dificultades destacan: "el ingreso al empleo estable y la cantidad de trabajo doméstico" (Ortiz-Ortega, 2013, p. 40), que se asume debe ser responsabilidad de la mujer. A estos se suma: "la fuerte predominancia masculina en la estructura de poder de la ciencia, que resulta en una caracterización de los méritos en la evaluación que corresponde a un ideal de trayectoria homogéneo y masculinizado" (Ortiz-Ortega, 2013, p. 40).

e) El reconocimiento como investigadoras (por ejemplo, su ingreso al Sistema Nacional de Investigadores -SNI- en México) muestra una participación desigual que se acrecienta a favor de los hombres, conforme se elevan los requerimientos para ascender en los niveles de estímulos. En 2010 "la mayoría de las investigadoras del SNI están en los niveles más bajos" [como candidatas o en nivel 1]. En el nivel de Eméritas (nivel 4) sólo tres mujeres alcanzaron esta distinción" (Ortiz-Ortega, 2013, pp. 41-42). 
Por su parte, Nash (2012) afirma que entre los factores que marcan la prevalencia de desigualdades sexo-genéricas en materia laboral se encuentra: "la elección del campo de estudio, los estereotipos [y] la demanda de horarios de trabajo más cortos o flexibles debido a la distribución desigual de los trabajos de cuidado" (p. 313). Con respecto de estos últimos se hace evidente que mientras las mujeres han ampliado su rol tradicional restringido de madre/ama de casa para incluir el de trabajadora y profesionista, en el caso de los hombres no se ha logrado un tránsito similar para incluir en su rol de trabajador/proveedor las tareas propias de los cuidados.

Los esfuerzos institucionales internacionales para promover la equidad de género son se materializan en avances legislativos significativos, políticas públicas que beneficien a las mujeres, por ello se reconoce que la cooperación y el intercambio internacional para promover la educación superior con lineamientos específicos sobre perspectiva de género son mecanismos decisivos que permiten tomar diversas acciones. Pero es necesario tener en cuenta que, en la práctica, algunos acuerdos o leyes no se ejecutan de manera adecuada a las propuestas es importante conocerlas ya que son elementos que fundamentan la lucha social y sientan las bases para lograr retos en beneficio social.

Dentro de la promoción de equidad de género, la educación superior a nivel mundial es un factor primordial. Al respecto destacan dos Conferencias Mundiales sobre la Educación Superior organizadas por la UNESCO (por sus siglas en inglés, Organización de las Naciones Unidas para la Educación, la Ciencia y la Cultura), dichas conferencias se realizaron en 1998 y 2009 en las que se evaluaron los avances y retos en este nivel.

En 1998 la Conferencia “La educación superior hacía en el siglo XXI. Visión y acción" celebrada en París, del 5 al 9 de octubre se planteó la necesidad de sacar este nivel educativo de la crisis en la que se encontraba inmersa. En uno de los artículos publicados se mencionó el "Fortalecimiento de la participación y promoción del acceso de las mujeres" en donde se plantean avances, pero se reconoce que en el acceso de las mujeres a la educación superior siguen existiendo obstáculos de índole, política, socioeconómica y cultural. Por lo anterior se propone consolidar la participación de las mujeres en todos los niveles y las disciplinas en las que no estén suficientemente representadas. 
En la Conferencia de 2009 se analizó como subtema la "Equidad, acceso y calidad" en el cual se discutieron con profundidad las situaciones de inequidad social que a diez años de la primera conferencia seguían persistiendo. A pesar de haberse incrementado el ingreso a la educación superior se recalcó el problema de que no todos los que ingresan logran terminar sus estudios. Se definió que la equidad no se logra sólo permitiendo el acceso a las instituciones de educación superior sino, sobre todo, asegurando la permanencia y el éxito escolar de los estudiantes. “Por consiguiente las instituciones de educación superior tienen la responsabilidad fundamental de lograr que los estudiantes que en ella se matriculan lleguen a graduarse" (UNESCO, 2009, s/p).

Pero, si bien los acuerdos internacionales junto con una férrea lucha feminista histórica han logrado que las mujeres accedan a la vida pública y exijan sus derechos la equidad de género no es aún una realidad. En materia de educación se observa que si bien ha aumentado su acceso a la educación superior aún queda por analizar el significado que la mujer otorga a sus estudios profesionales, al respecto Bonder plantea:

De todas maneras, y como en otros niveles de la enseñanza, uno de los problemas fundamentales que aún persisten es la orientación que dan a sus estudios y cuál es el sentido y el impacto de su paso por la universidad en la determinación de su estilo de vida y en su experiencia familiar y laboral futura. Las informaciones regionales revelan que las mujeres optan preferentemente por las áreas correspondientes a educación, bellas artes y ciencias sociales (2004, p.21).

Y aunque lo anterior se planteó hace más de 20 años sigue siendo una constante encontrar una matrícula mayor de mujeres en disciplinas enfocadas a las humanidades frente a una escasa participación en las ciencias consideradas como "duras" (a nivel de licenciatura y posgrado); y esta participación no se limita al rol de estudiantes sino al de docentes e investigadoras. La escasa participación de mujeres en los núcleos académicos de los posgrados analizados en esta investigación, los cuales se ubican en el campo de las ciencias naturales o las ingenierías, es muestra de esta realidad. Además, si en las ciencias sociales y humanidades las mujeres han logrado importantes espacios, su participación en los espacios de dirección de más alto nivel y toma de decisiones, sigue siendo menor. 
Para atender esta inequidad, los Objetivos de Desarrollo del Milenio (ODM) de la Organización de las Naciones Unidas (ONU) plantean, en su objetivo número 3, plantea la igualdad entre los sexos y el empoderamiento de la mujer "No habrá posibilidad de que la pobreza pase a la historia si no se mejora significativa y rápidamente la vida de las mujeres y las niñas en todos los países" (OCDE, 2013, s/p), en cuya meta se encuentra eliminar las desigualdades a través del desarrollo de la enseñanza en todos los niveles y aunque homogenizar los niveles educativos a nivel mundial estaba programada para el 2015 fue una de las asignaturas no cumplidas.

Aunque en las últimas décadas se ha registrado una mayor participación laboral de las mujeres, estas trabajan por lo general en la parte informal de los mercados laborales, con pocas ganancias y condiciones laborales inseguras, lo que multiplica el riesgo de pobreza a lo largo de su vida y reduce la probabilidad de que disfruten de la cobertura de los sistemas de protección social o de prestaciones tales como las pensiones de jubilación. Las mujeres aún se enfrentan a una brecha de remuneración por razón de sexo, a la segregación en ciertas profesiones y a techos de cristal, por lo que están sobre-representadas en los puestos de trabajo mal pagados e infrarepresentadas en los puestos superiores (OCDE, 2013, s/p).

Lo anterior con respecto de atender a las necesidades laborales, pero únicamente se podrá lograr esto, con la calidad educativa adecuada que responda a las necesidades de todos y todas, pero especialmente de las mujeres, por tanto, es importante mencionar la falta de oportunidades en los sectores de Ciencia y Tecnología.

\section{Trabajo descriptivo}

Ahora bien, con lo descrito en el apartado anterior podemos dar cuenta de las desigualdades que aún prevalecen en los espacios que ocupan las académicas, ahora nos situaremos en las IES de Chiapas a través de un análisis descriptivo, los resultados que arrojó este primer paso de la investigación fueron reveladores que dan cuenta de la brecha 


\section{ARTÍCULOS}

de género que prevalece a la actualidad, cabe señalar que los datos que se analizan son de la convocatoria 2019 del SNI del CONACyT ${ }^{1}$.

\section{Universidad Autónoma de Chiapas (UNACH)}

La Universidad Autónoma de Chiapas (UNACH) es una institución de educación superior pública que fue fundada en Tuxtla Gutiérrez, Chiapas; en 1975 instaurada por el entonces gobernador del estado Manuel Velasco Suárez. Actualmente esta universidad tiene su sede en Tuxtla Gutiérrez pero cuenta con otros espacios académicos como escuelas, centros, institutos y facultades agrupadas en campus en diversos municipios del estado que por lo general pretenden atender las necesidades de cada una de las regiones donde se encuentren, a la fecha cuenta con nueve campus.

Con respecto de la UNACH se encontraron un total de 148 docentes pertenecientes al SNI, de los cuales 102 son hombres, es decir el 69 por ciento y 46 son mujeres, lo que da como resultado que el 31 por ciento son académicas; los datos por si solos ya nos revelan las diferencias marcadas y se puede notar la brecha de género existente.

Gráfica 1: Académicas y académicos UNACH

\section{SNI-UNACH}

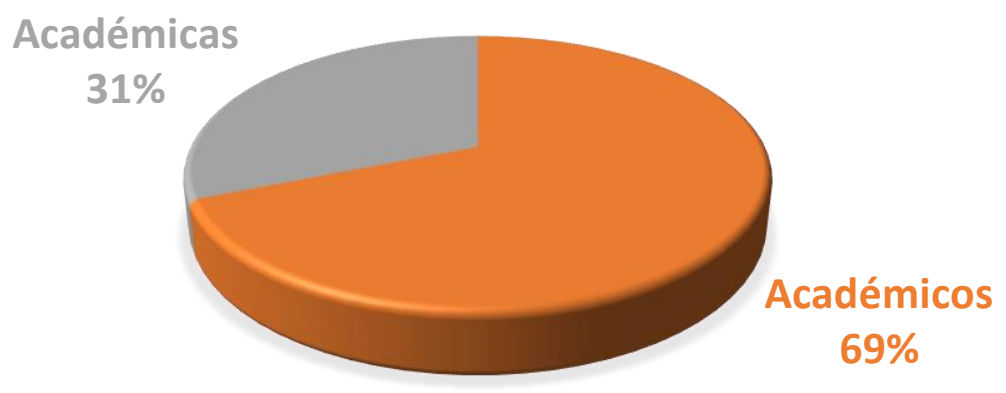

${ }^{1}$ Se analizan los datos de esa convocatoria, ya que fue a inicios de noviembre de 2020 cuando se emitieron los resultados de la convocatoria 2020. 


\section{ARTÍCULOS}

\section{Universidad de Ciencias y Artes de Chiapas (UNICACH)}

La Universidad de Ciencias y Artes de Chiapas (UNICACH) representa una fusión del Instituto de Ciencias y Artes de Chiapas (ICACH) con el Instituto Chiapaneco de Cultura (ICHC), instituciones con amplio reconocimiento a lo largo de la historia de la educación en Chiapas. Esta universidad es un organismo descentralizado autónomo de administración pública.

Por su parte la UNICACH cuenta con un total de 86 integrantes del SNI de los cuales 51 son hombres, lo que arroja un 60 por ciento de presencia de investigadores y por su parte son 35 mujeres las que pertenecen a este padrón, lo que da como resultado un 40 por ciento de participación de académicas.

Gráfica 2: Académicas y académicos de la UNICACH

\section{SNI-UNICACH}

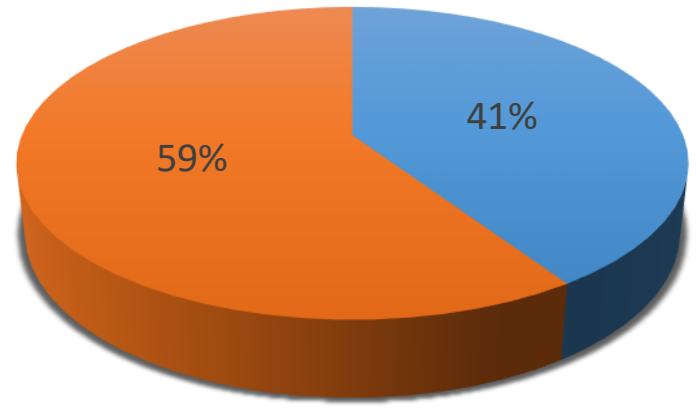

\section{Centro de investigaciones y Estudios Superiores en Antropología Social (CIESAS)}

De acuerdo con la historia de creación del Centro de Investigaciones y Estudios Superiores en Antropología Social (CIESAS) esta IES fue fundada en septiembre de 1973 como Centro de Investigaciones Superiores del Instituto Nacional de Antropología e Historia (CISINAH). Haciendo una reestructuración en 1980 se definió como CIESAS, actualmente 


\section{ARTÍCULOS}

más amplio de las áreas disciplinares que se ofertan en estas universidades, porque se constatado en anteriores investigaciones que las correspondencias de las áreas disciplinares también es un factor a considerar.

Gráfica 4: académicas y académicos ECOSUR

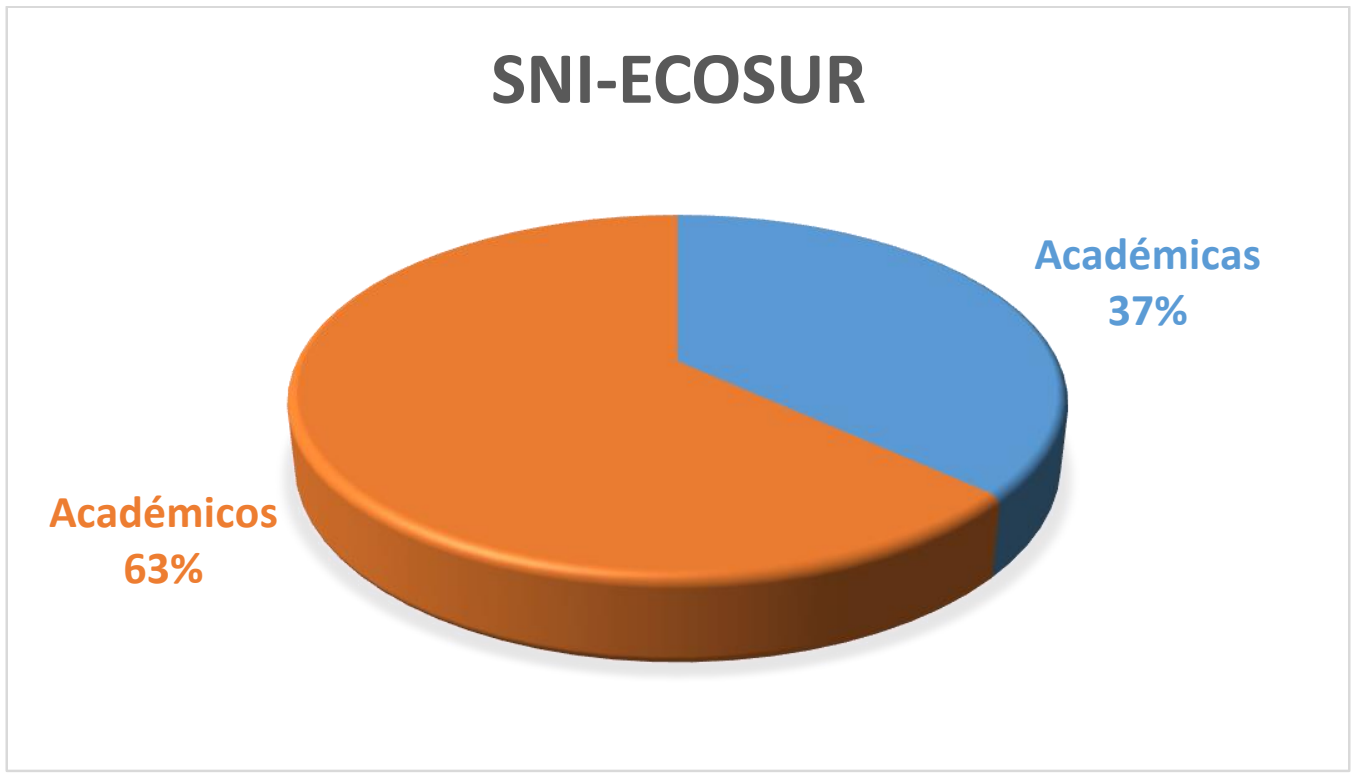

\section{Instituto Tecnológico de Tuxtla Gutiérrez (ITTG)}

De acuerdo con la página web oficial del Instituto Tecnológico de Tuxtla Gutiérrez (ITTG), es en la década de 1970 cuando se crea del Instituto Tecnológico Regional de Tuxtla Gutiérrez (ITRTG) hoy Instituto Tecnológico de Tuxtla Gutiérrez (ITTG). En 1971 se colocó la primera piedra del proyecto y en octubre de 1972 ya se contaba con infraestructura suficiente para abrir sus puertas en Tuxtla Gutiérrez con las carreras de Técnico en Máquinas de Combustión Interna, Electricidad, Laboratorista Químico y Máquinas y Herramientas. Posteriormente, en 1974 inició con la modalidad en el nivel superior, ofreciendo las carreras de Ingeniería Industrial en Producción y Bioquímica en Productos Naturales. En 1980 se amplió la oferta educativa al incorporarse las carreras de Ingeniería Industrial Eléctrica e Ingeniería Industrial Química.

Para esta universidad se identifica 5 académicas y 17 académicos de manera porcentual encontramos el 23 por ciento y 77 por ciento respectivamente, aquí se acentúa mucho más las diferencias existentes, otro de los elementos a considerar, como ya se había

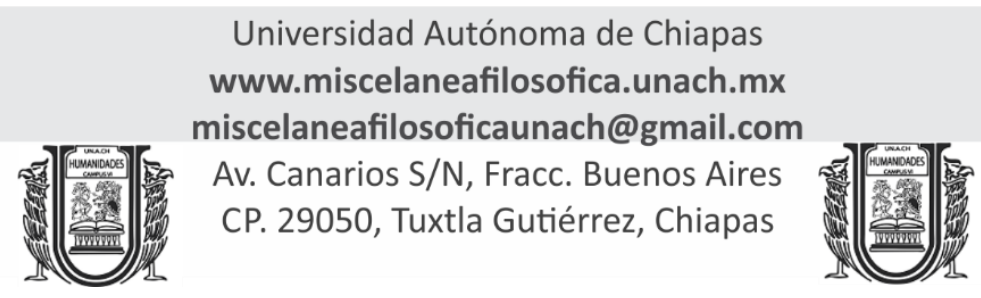


Gráfica 7: Total de académicos y académicas en el SNI en Chiapas.

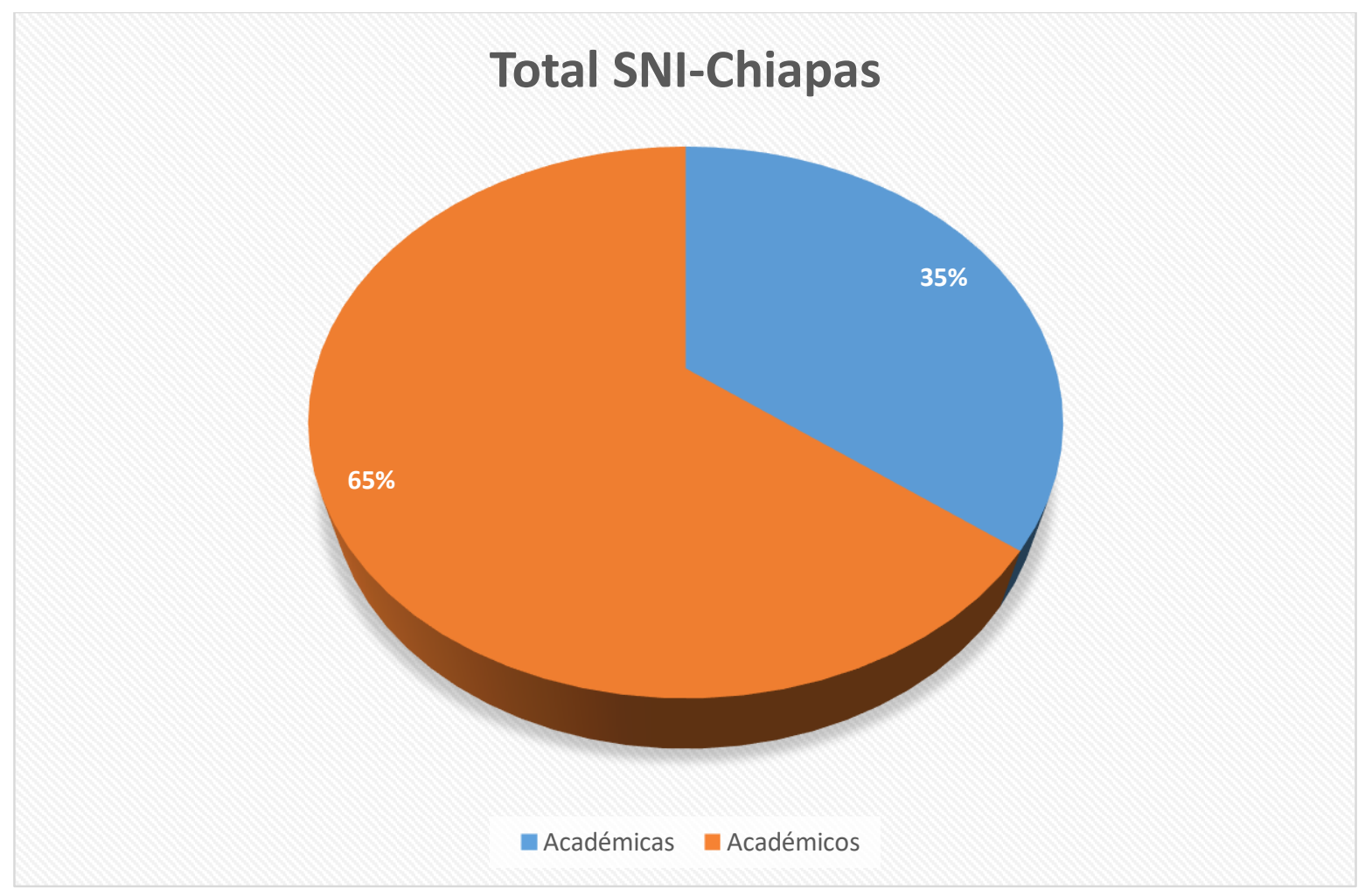

\section{CONCLUSIONES}

Podemos observar una inequidad visible en la en la inclusión de las académicas en este sistema nacional que fue creado en 1984 con la intención de reconocer la labor de las y los investigadores que se dedican a la producción del conocimiento científico y tecnológico del país, cabe destacar que este reconocimiento se da a través de una evaluación rigurosa entre pares, dicha distinción simboliza de cierta forma calidad y prestigio a nivel nacional, además de ser un reconocimiento económico al que en la mayoría de casos (aquellos que tienen adscripción institucional) logran acceder, cabe destacar que además que este reconocimiento se otorga con categorías que van del menor al mayor reconocimiento, entre los que se encuentran candidato/a, nivel 1, nivel 2 y nivel 3. Es por tanto que logran acceder a este sistema es sumamente complejo y lo que simboliza de cierta forma es la consolidación en la investigación. 
